\title{
Ultrastructural and Histopathological Studies of 2009 Pandemic Influenza A (H1N1) Virus
}

Cynthia S. Goldsmith ${ }^{1}$, Maureen G. Metcalfe ${ }^{1}$, Wun-Ju Shieh ${ }^{1}$, Dianna M. Blau ${ }^{1}$, Dominique C. Rollin ${ }^{1}$, Xiyan $\mathrm{Xu}^{2}$, and Sherif R. Zaki ${ }^{1}$.

${ }^{1}$ Infectious Disease Pathology Branch, National Center for Emerging and Zoonotic Infectious Diseases (proposed), and ${ }^{2}$ National Center for Immunization and Respiratory Diseases, Centers for Disease Control and Prevention (CDC), Atlanta, GA 30333

In April 2009, a novel H1N1 influenza virus was first detected in 2 patients in California, and the same virus was found to be circulating in Mexico. The virus rapidly spread worldwide, and by June, the World Health Organization had declared a global pandemic. The virus, previously called swine flu, contains a unique combination of gene segments from both North American and Eurasian swine lineage, and became known as 2009 pandemic influenza A (H1N1) virus. Symptoms of infection included cough, fever, and shortness of breath. Most cases were fairly mild, although there was a mortality rate of $0.05 \%$ in the US.

The ultrastructural characteristics of influenza viruses have been well documented [1]. In viruses grown in cell culture, as seen in Fig. 1, the hemagglutinin and neuraminidase glycoproteins form prominent protrusions (spikes) from the viral envelope. Inside the virions are 8 individual nucleocapsids, which contain the RNA of the virus wrapped within a nucleoprotein. Negative stain images of the virus particles show enveloped virions with well-defined spikes (Fig. 2). Influenza virions are known to form upon the apical plasma membrane in tissue culture cells that are polarized. However, in 2009 pandemic influenza (H1N1) virus infection, not only are virions budding at the plasma membrane, but also upon the membranes of the Golgi complex (Fig. 3). Similar budding patterns have been noted in cells infected with Influenza A (H5N1), also known as "avian influenza." Dark tubular structures can be found in the nuclei of infected cells, and by using immunogold labeling these tubules were shown to contain the matrix (M) protein (Fig 4). The tubular structures can also be found in autopsy tissue (Fig. 5), and in cell cultures infected with other influenza A viruses. Electron microscopy of autopsy lung tissue revealed characteristic extracellular influenza virus particles (Fig 6).

Histopathological examination of lungs from autopsy tissues found evidence of diffuse alveolar damage (DAD), including inflammation, edema, and hyaline membranes (Fig. 7) [2]. Immunohistochemical testing detected influenza antigens in the pneumocytes in the alveoli, in epithelial cells in the airways, and in mucous glands.

2009 pandemic influenza A (H1N1) virus was the causative agent of the first influenza pandemic since 1968. Morphological and pathological studies are instrumental in the understanding of this novel virus, and may provide information for treatment and vaccine development.

References

[1] PW Choppin, et al., The Influenza Viruses and Influenza (1975) 12.

[2] WJ Shieh, et al., submitted for publication. 

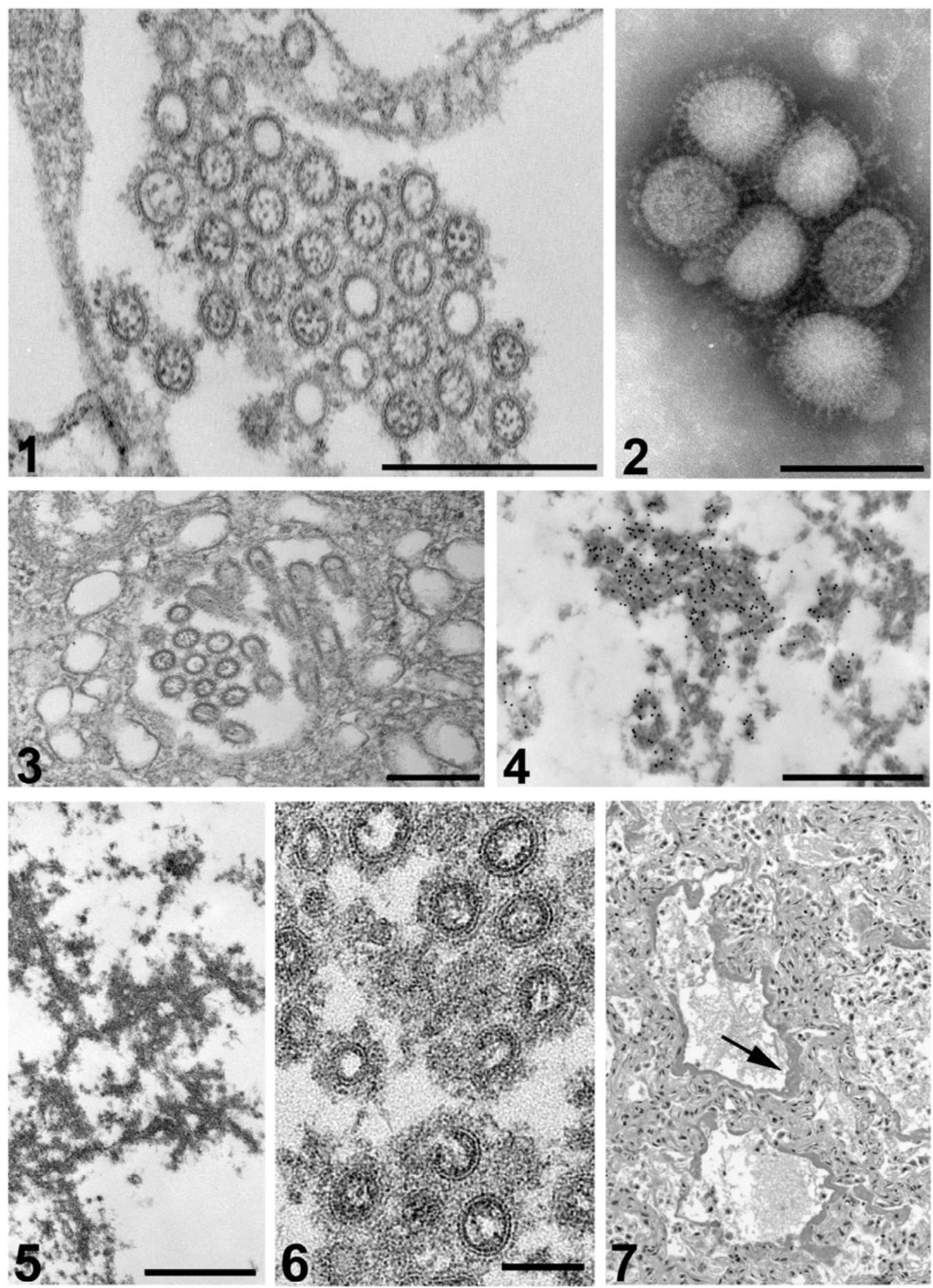

Fig. 1. Extracellular influenza virus particles showing internal nucleocapsids, seen in cross section, surrounded by an envelope with prominent surface projections. Bar, $300 \mathrm{~nm}$. Fig. 2. Negatively stained virions. Bar, $140 \mathrm{~nm}$. Fig. 3. Virus particles budding into the Golgi complex. Bar, $300 \mathrm{~nm}$. Fig. 4. Immunogold labeling of the $M$ protein on tubular structures in the nucleus of an infected tissue culture cell cell. Bar, $700 \mathrm{~nm}$. Fig. 5. Dark tubules in nucleus of alveolar cell in human autopsy tissue. Bar, $100 \mathrm{~nm}$. Fig. 6. Extracellular influenza virus particles found with a damaged cell in the alveolus. Bar, $100 \mathrm{~nm}$. Fig. 7. Diffuse alveolar damage in lung tissue, with hyaline membranes (arrow) lining the alveolar space. Hematoxylin-eosin stain. 